\title{
FOLIATIONS AND NONCOMPACT TRANSFORMATION GROUPS
}

\author{
BY MORRIS W. HIRSCH
}

Communicated by Stephen Smale, November 20, 1969

Introduction. Let $G$ be a Lie group and $M$ a compact $C^{\infty}$ manifold. In [2] Anosov actions of $G$ on $M$ are defined and proved to be structurally stable.

In this announcement we are concerned with the foliation $\mathscr{F}$ of $M$ defined by the orbits of $G$. Under the assumption that $G$ is connected, $\mathcal{F}$ is $C^{1}$ stable (3). If $G$ is connected and nilpotent, $G$ has a compact orbit (4). If $G$ is merely solvable, however, there may be no compact orbit. In fact it can happen that no foliation $C^{0}$ close to $\mathfrak{F}$ has a compact leaf (8). Upper bounds for the number of compact orbits of given type are found (9). In (7) we discuss the intersection of certain nilpotent subgroups of a Lie group $S$ with conjugates of a uniform discrete subgroup of $S$.

Hyperbolic automorphisms of foliations. A $k$-foliation $\mathfrak{F}$ of $M$ is a function assigning to each $x \in M$ the image $\mathfrak{F}_{x}$ of a $C^{2}$ injective immersion $V_{x} \rightarrow M$ of a connected $k$-dimensional manifold $V_{x}$. We require that the leaf $\mathcal{F}_{x}$ contain $x$, and that the function $T \mathfrak{F}: M \rightarrow G_{k}(M)$ assigning to $x \in M$ the tangent plane to $V_{x}$ at $x$ be $C^{1}$; here $G_{k}(M)$ is the manifold of $k$-planes tangent to $M$. Equivalently, $T$ F is a completely integrable $C^{1}$ field of $k$-planes, and $\mathcal{F}_{x}$ is the maximal integral submanifold through $x$. Thus $\left\{\mathcal{F}_{x}\right\}_{x \in M}$ is a partition of $M$. The set $F_{k}(M)$ of all $k$-foliations of $M$ inherits the $C^{0}$ and $C^{1}$ topologies from the set of $C^{1}$ maps $M \rightarrow G_{k}(M)$. We also use $T$ F to denote the bundle of $k$-planes tangent to the leaves.

If $\mathcal{F}, \mathcal{G} \in F_{k}(M)$, a homeomorphism $h: \mathcal{F} \rightarrow \mathcal{G}$ is a homeomorphism of $M$ taking each leaf of $\mathfrak{F}$ onto a leaf of $\mathcal{G}$. We call $\mathfrak{F} C^{1}$ stable if it has a $C^{1}$ neighborhood $N \subset F_{k}(M)$ of foliations homeomorphic to $\mathscr{F}$.

An automorphism $g$ of $\mathcal{F}$ is a $C^{1}$ diffeomorphism of $M$ which is a homeomorphism $\mathfrak{F} \rightarrow \mathfrak{F}$. We call $g$ hyperbolic if there exists a splitting $T M=E_{+} \oplus E_{-} \oplus T \mathcal{F}$ invariant under $T g$, and such that the following condition holds. For some (and hence any) Riemannian metric on $M$ there exist constants $0<\lambda<1<\mu$ and $n \in Z_{+}$such that if $X \in T M$ and $X \neq 0$, then

AMS 1969 subiect classifications. Primary 2240, 5736; Secondary 5482, 3465, $3451,3453$.

Key words and phrases. Foliation, transformation group, compact orbit, structural stability. 


$$
\begin{array}{ll}
\quad\left|T g^{n} X\right|<\lambda|X| & \text { if } X \in E_{-} ; \\
\mu|X|<\left|T g^{n} X\right| & \text { if } X \in E_{+} ; \\
\mu|X|>\left|T g^{n} X\right|>\lambda|X| & \text { if } X \in T F .
\end{array}
$$

A deformation of $F$ is an automorphism $g$ which is homotopic to the identity by a homotopy $g_{t}$ such that $g_{t}\left(F_{x}\right) \subset F_{x}$ for all $x \in M$ and $0 \leqq t \leqq 1$.

(1) Theorem. A foliation of $M$ which admits a hyperbolic deformation is $C^{1}$ stable.

The proof uses the stable manifold theory of [2].

(2) TheOREM. Let $g$ be a deformation of $\mathfrak{F} \in F_{k}(M)$ such that for some Riemannian metric on $M$, the Jacobian of

$$
T g \mid T F_{x}: T F_{x} \rightarrow T F_{g x}
$$

is $\neq 1$ for all $x$. Then $\mathfrak{F}$ has a $C^{0}$ neighborhood $N \subset F_{k}(M)$ such that no element of $\boldsymbol{N}$ has a compact leaf.

The idea of the proof is that if $\mathcal{G} \in N$, some approximation to $g$ will be an automorphism of $\mathcal{G}$ which preserves every leaf of $g$ but changes its volume.

Anosov actions. Let $G$ be a Lie group acting differentiably of class $C^{2}$ on $M$. Suppose the action is locally free, i.e., every isotropy group is discrete. Then the components of orbits define a foliation $\mathcal{F}$, and every $g \in G$ is an automorphism of $\mathcal{F}$. If $g$ is a hyperbolic automorphism, we call $g$ an Anosov element. If an Anosov element exists we call the action an $A$ nosov action; and we also say $G$ acts hyperbolically.

Let $G_{1}$ be the identity component of $G$.

Let $\mathfrak{F}$ denote the orbit foliation of an Anosov action of $G$ on $M$.

(3) Theorem. If $G / G_{1}$ is finite, or if $G_{1}$ contains an Anosov element, then $\mathfrak{F}$ is $C^{1}$ stable.

Proof. Follows from (1).

(4) Theorem. Assume $G$ is nilpotent and $G / G_{1}$ finite. Then $G$ has a compact orbit. In fact if $A \subset G$ is a 1-parameter subgroup containing an Anosov element $g$, the nonwandering set of the $A$ flow on $M$ lies in the closure $K$ of the union of the compact $G$ orbits. If $g$ is measure preserving on $M$, then $M=K$, and some orbit is dense.

The proof uses [2] to obtain a subgroup $H \subset G$ containing an Anosov element, such that $H(p)$ is compact for some $p \in M$. Since $G$ 
is nilpotent, the normalizer $N$ of $H$ is bigger than $H$, and [2] is used to prove that $N_{1}(p)$ is compact. By taking successive normalizers we arrive at $G$.

\section{Examples.}

(5) EXAMPLE. If $G$ acts hyperbolically on $M$ and $H$ acts locally freely and transitively on $N$, then $G \times H$ acts hyperbolically on $M \times N$.

(6) ExAmpLE. Let $G$ be an analytic subgroup of a Lie group $S$. Call $G$ hyperbolically embedded if for some $X \in \mathcal{L}(G)$ (the Lie algebra of $G$ ), $\operatorname{ad}_{G} X$ has its spectrum on the imaginary axis, while $\operatorname{ad}_{S} X$ induces an endomorphism of $\mathscr{L}(S) / \mathscr{L}(G)$ having no spectrum on the imaginary axis. If $\Gamma \subset S$ is a uniform discrete subgroup, then $G$ acts hyperbolically on $S / \Gamma$. For example, $S=S L(n, R)$ and $G$ is the diagonal subgroup. Note that Borel [1] proved that every semisimple Lie group has a uniform discrete subgroup.

From (4) we obtain

(7) THEOREM. Let $G$ be a connected nilpotent group which is hyperbolically embedded in $S$. Let $\Gamma \subset S$ be a uniform discrete subgroup. Then the set $\left\{s \in S \mid G / G \cap s \Gamma s^{-1}\right.$ is compact $\}$ is dense in $S$; thus $G$ has a uniform discrete subgroup.

(8) Example. Let $A$ be a $2 \times 2$ real matrix such that $e^{A}$ has integer entires, and its eigenvalues are $\lambda$ and $\lambda^{-1}, 0<\lambda<1$. Let $R$ act on $R^{2}$ by $t(x)=e^{t A}(x)$. Let $S$ be the semidirect product $R^{2} \cdot R$. Note that $\boldsymbol{Z} \subset \boldsymbol{R}$ leaves $\boldsymbol{Z}^{2} \subset R^{2}$ invariant, and set $\Gamma=Z^{2} \cdot \boldsymbol{Z} \subset R^{2} \cdot R$. Put $M^{3}=S / \Gamma$; then $M^{3}$ is compact. Topologically, $M^{3}$ is obtained from $\left(R^{2} / Z^{2}\right) \times I$ by identifying $\left(x+Z^{2}\right) \times 0$ with $\left(e^{A}(x)+Z^{2}\right) \times 1$. Let $L \subset R^{2}$ be the $\lambda$ eigenspace of $e^{A}$ and let $G=L \cdot R \subset R^{2} \cdot R$. Then $G$ is a solvable nonnilpotent 2-dimensional Lie group acting hyperbolically on $M^{3}$. The element $(0,1) \in G$ is an Anosov element. It follows from Theorem 2 that no sufficiently small $C^{0}$ perturbation of the orbit foliation of $G$ on $S / \Gamma$ has a compact leaf. More generally, this is the case if $G$ is hyperbolically embedded in $S$ as in (6), and $\operatorname{tr}\left(\operatorname{ad}_{G} X\right)$ $\neq 0$.

In contrast to this phenomenon, Roussarie and Weil state that any locally free action of $R^{2}$ can be $C^{0}$ approximated by an action having compact orbits; see [4].

Counting compact orbits. Fix a Haar measure $m$ for $G$. If $\Gamma \subset G$ is a discrete subgroup such that $G / \Gamma$ is compact, it is well known that $G$ is unimodular. Hence $\Delta(\Gamma)$ depends only on the conjugacy class $[\Gamma]$ of $\Gamma$, where $\Delta(\Gamma)$ is the measure of $G / \Gamma$ induced by $m$. 
Let $G$ act on $M$. The type of an orbit is the conjugacy class of the isotropy group of any of its elements. Let $N(\Gamma)$ be the number of compact orbits of type $[\Gamma]$.

(9) Theorem. Given an Anosov action of $G$ on $M$, there is a constant $B$ such that $N(\Gamma) \leqq B^{\Delta(\Gamma)}$ for every uniform discrete subgroup $\Gamma \subset G$.

This theorem generalizes results of K. Meyer [3] and M. Shub [5]. The proof imitates Shub's.

\section{BiBLIOGRAPHY}

1. A. Borel, Compact Clifford-Klein forms of symmetric spaces, Topology 2 (1963), 111-122. MR 26 \#3823.

2. M. Hirsch, C. Pugh and M. Shub, Invariant manifolds, Bull. Amer. Math. Soc. 76 (1970), 1015-1019.

3. K. R. Meyer, Periodic points of diffeomorphisms, Bull. Amer. Math. Soc. 73 (1967), 615-617. MR 36 \#4573.

4. H. Rosenberg, R. Roussarie, and D. Weil, A classification of closed orientable 3manifolds of rank two, University of Paris, Orsay. (mimeographed) (1968).

5. M. Shub, Periodic orbits of hyperbolic diffeomorphisms and flows, Bull. Amer. Math. Soc. 75 (1969), 57-58. MR 38 \#2815.

University of California, Berkeley, California 94720. 